\title{
Effects of Milk Casein Derived Tripeptides on Endothelial Enzymes In Vitro; a Study with Synthetic Tripeptides
}

Authors

Affiliation

\author{
A. Siltari, A. S. Kivimäki, P. I. Ehlers, R. Korpela, H. Vapaatalo
}

Institute of Biomedicine, Pharmacology, University of Helsinki, Helsinki, Finland

\author{
Key words \\ - blood pressure \\ renin-angiotensin-system \\ - endothelium \\ - tripeptide \\ - cathepsin G \\ prolyl oligopeptidase
}

received 06.03 .2012 accepted 04.07.2012

\section{Bibliography DOI http://dx.doi.org/ 10.1055/s-0032-1321846 Published online: August 23, 2012 Arzneimittelforschung 2012; 62: 477-481 \\ (c) Georg Thieme Verlag KG Stuttgart · New York ISSN 0004-4172}

Correspondence

\section{A. Siltari}

Institute of Biomedicine, Pharmacology University of Helsinki P. O. Box 63, 00014 University of Helsinki Finland

Tel.: + 358/50/544 8227

Fax: $+358 / 191 / 25364$

aino.siltari@helsinki.fi

\section{Abstract \\ $\nabla$}

In the fermentation of milk by certain lactic acid bacteria, casein is degraded into bioactive tripeptides shown to lower blood pressure in experimental animal models and in mildly hypertensive humans. This effect is suggested to result mainly in inhibition of angiotensin converting enzyme 1 (ACE-1).

Due to the complexity of renin-angiotensin system (RAS), several other enzymes than ACE- 1 can participate in the production of vasoactive components. Therefore, in the present study we investigated effects of tripeptides isoleucine-proline-proline (IPP), valine-proline-proline (VPP) and leucine-proline-proline (LPP) on some endothelial enzymes that are important in RAS or oth-

\section{Introduction}

$\nabla$

Blood pressure and vascular functions are regulated by numerous circulating and local factors. The main regulatory system is renin-angiotensin system (RAS) ( $\bullet$ Fig. 1), but also other endothelial enzymes produce highly active compounds such as prostacyclin and nitric oxide. The most biologically active substance in RAS is angiotensin II (Ang II), which is broken down from angiotensin I (Ang I) mainly by angiotensin converting enzyme I (ACE-1) but also by chymase and cathepsin G. ACE-1 also degrades bradykinin, a vasodilatory peptide into inactive fragments ( $\bullet$ Fig. 1). RAS contains a number of other enzymes besides ACE-1 which have an important role in blood pressure regulation. Some of these enzymes have a direct effect on Ang II but they also participate in the conversion of substances such as angiotensin-(1-7) (Ang-(1-7)) with potent vasodilatory and cardioprotective effects. [1-3] In addition to classical AT-1 receptors, new focus has also been set to vasodilatory AT-type 2 and Ang-(1-7)-Mas- erwise have a role in the endothelial function. The enzymes investigated were renin, chymase, neutral endopeptidase (NEP), prolyl oligopeptidase (POP), cathepsin G, endothelin converting enzyme 1 (ECE-1), and cyclooxygenase 1 and 2 (COX -1 and COX-2).

The tripeptides inhibited prolyl oligopeptidase (POP) dose-dependently. IPP was the most potent inhibitor ( $\left.\mathrm{IC}_{50} 486 \pm 95 \mu \mathrm{M}\right)$. Contrary, cathepsin $G$ was activated by IPP, VPP and LPP as well as the amino acids proline and isoleucine. The other investigated enzymes were not affected. Inhibition of POP and activation of cathepsin G do not explain the blood pressure lowering effects of the tripeptides. Thus the inhibition of ACE- 1 remains the most plausible mechanism of the antihypertensive effects of the tripeptides. receptors [3,4]. Ang-(1-7) also increases the physiological impact of bradykinin. [3-5]

When milk is fermented e.g. with $L$. helveticus or S. cerevisiae, casein is degraded into bioactive tripeptides isoleucine-proline-proline (IPP), leucine-proline-proline (LPP) and valine-prolineproline (VPP). These tripeptides have been found to lower blood pressure and improve vascular function in hypertensive rats [6-9] and in humans [10-12]. Their blood pressure-lowering effect is believed to result mainly from the inhibition of ACE- 1 activity $[6,13]$, but some of their favorable vascular functions are different from those of captopril, a standard ACE inhibitor [8]. This could be related e.g. to inhibition of arginase [13] which increases local substrate concentration for nitric oxide production.

The effects of the casein derived tripeptides cannot be explained solely by ACE- 1 inhibition due to their manifold pharmacological effects. Therefore we investigated their effects on other blood pressure and vascular function regulating enzymes in vitro. 


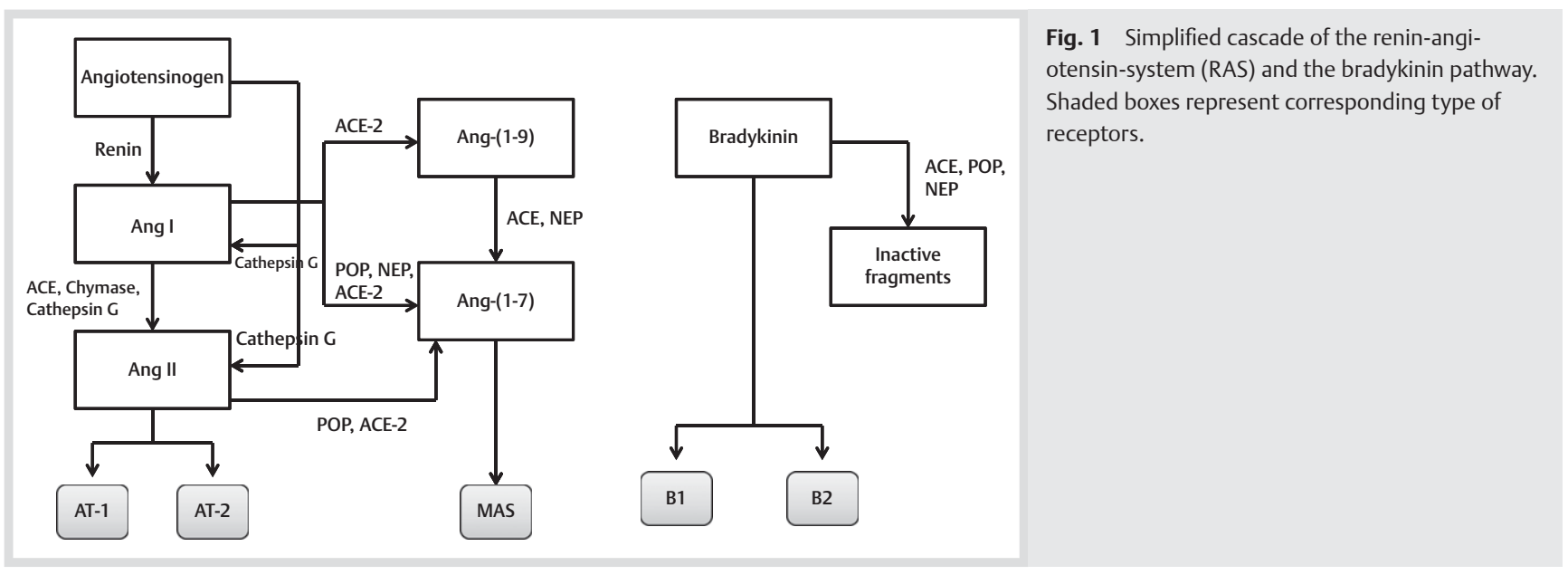

\section{Materials and methods}

Tripeptides IPP, VPP and LPP, substrates dansyl-D-Ala-Gly-4-Nitro-Phe-Gly-OH (DAGNPG), Z-Gly-Pro-AMC and N-Suc-Ala-AlaPro-Phe-pNA were purchased from Bachem (Weil am Rein, Germany). NEP was purchased from Innovative Research (Novi, MI, USA). POP (Recombinant Human), ECE-1 (Recombinant Human) and MCA-Arg-Pro-Pro-Gly-Phe-Ser-Ala-Phe-Lys-(DNP) $\mathrm{OH}$ were purchased from R\&D Systems (Minneapolis, MO, USA). Cathepsin G (Human neutrophil) was purchased from Merck Chemicals (Nottingham, UK).

\section{Renin assay}

Renin (Human recombinant) activity was measured using a commercial fluorometric kit (SensoLyte ${ }^{\circledR} 520$ Renin Assay Kit, AnaSpec, San Jose, CA, USA). The activity of renin was determined using 5-FAM/QXL ${ }^{\mathrm{TM}} 520$ fluorescence resonance energy transfer (FRET) peptide as a substrate which renin degrades into 2 separate fragments. The fluorescent can be monitored at ex/ em $=490 / 520 \mathrm{~nm}$ (Perkin Elmer Life Science, WALLAC VICTOR ${ }^{2}$ 1420, Turku; Finland). Incubation time was $60 \mathrm{~min}$ at $37^{\circ} \mathrm{C}$ including 11 fluorescent measurements with all the tripeptides concentrations $(1 \mu \mathrm{M}-3.3 \mathrm{mM})$. The tripeptides were solved and diluted in the assay buffer.

\section{Chymase assay}

Chymase (Human recombinant) activity was measured using commercial kit (Chymase activity kit, Sigma-Aldrich, Jerusalem, Israel). The method is based on the ability of chymase to degrade N-Succinyl-Ala-Ala-Pro-Phe-p-nitroanilide into a colorimetric product which can be monitored at wavelength $405 \mathrm{~nm}$ (Multiscan RC, Labsystems, Helsinki, Finland). Incubation time was 50 min at $37^{\circ} \mathrm{C}$ including 9 measurements with all tripeptides concentrations $(1 \mu \mathrm{M}-1 \mathrm{mM})$. The tripeptides were solved and diluted in the assay buffer.

\section{Neutral endopeptidase (NEP) assay}

NEP activity was measured using the method by Florentin et al. [14] with small modifications. Assay substrate was DAGNPG which was diluted first in methanol and thereafter in assay buffer Tris- $\mathrm{HCl}(50 \mathrm{mM}, \mathrm{pH} 7.4)$, the final concentration of the substrate in the reaction was $50 \mu \mathrm{M}$. Methanol concentration in the reaction was $0.2 \%$. The method was based on NEPs ability to degrade DAGNPG of which fluorescense was monitored at ex/ em $=342 / 580 \mathrm{~nm}$ (Perkin Elmer Life Science, WALLAC VICTOR ${ }^{2}$
1420). Incubation time was $120 \mathrm{~min}$ at $37^{\circ} \mathrm{C}$ including 7 measurements with all tripeptides concentrations $(1 \mu \mathrm{M}-1 \mathrm{mM})$. The tripeptides and the enzyme were solved and diluted in the assay buffer.

\section{Prolyl oligopeptidase (POP) assay}

POP activity was measured using the method design by Stanziola et al. [15] and Atack et al. [16]. Assay substrate was Z-GlyPro-AMC from which POP can cleave AMC group, a fluorescent substance which can be measured in ex/em $=355 / 460 \mathrm{~nm}$ (Perkin Elmer Life Science, WALLAC VICTOR ${ }^{2} 1420$ ). The assay buffer was Tris $(25 \mathrm{mM})-\mathrm{NaCl}(250 \mathrm{mM})$ - dithiotreitol (DTT, $2.5 \mathrm{mM}$ ) buffer ( $\mathrm{pH} 7.5$ ). The enzyme, the tripeptides and the substrate were diluted in the assay buffer. The final concentrations of the enzyme, the substrate and the tripeptides in reaction were $0.005 \mu \mathrm{g}, 50 \mu \mathrm{M}$ and $1 \mu \mathrm{M}-3.3 \mathrm{mM}$, respectively, and the incubation time was $60 \mathrm{~min}$, including 10 measurements at room temperature.

\section{Cathepsin $\mathrm{G}$ assay}

Cathepsin G activity was measured using the method by Barret [17] with a small modifications. The method is based on the ability of cathepsin G to degrade the substrate N-Suc-Ala-AlaPro-Phe-pNA into a colorimetric product that can be measured spectrophotometrically at $410 \mathrm{~nm}$ (Multiscan RC, Labsystems, Helsinki, Finland). The substrate was diluted in dimethyl sulfoxide (DMSO). The final concentration of the substrate and DMSO in the reaction was $200 \mu \mathrm{M}$ and $25 \%$, respectively. The enzyme (0.1-0.2 mU/reaction) was diluted in ice cold distilled water. The tripeptides were solved and diluted in HEPES-NaOH buffer (100 mM, pH 7.5) which was the assay buffer. The incubation time was $70 \mathrm{~min}$ at $37^{\circ} \mathrm{C}$ including 11 measurements with all tripeptide concentrations $(0.1 \mu \mathrm{M}-3.3 \mathrm{mM})$.

\section{Endothelin converting enzyme I (ECE-1) assay}

ECE-1 activity was measured using Activity Assay Protocol by $\mathrm{R} \& \mathrm{D}$ systems. The assay procedure is based on the ability of ECE-1 to degrade MCA-Arg-Pro-Pro-Gly-Phe-Ser-Ala-Phe-Lys(DNP)OH and the development of the fluorescence products can be measured in ex/em $=320 / 405 \mathrm{~nm}$ (Perkin Elmer Life Science, WALLAC VICTOR $^{2} 1420$ ). The assay buffer was $0.1 \mathrm{mM} 2$-(N-morpholino)ethanesulfonic acid (MES) - $0.1 \mathrm{mM} \mathrm{NaCl}$-buffer (pH 6). The substrate $(10 \mu \mathrm{M})$, the enzyme $(0.005 \mu \mathrm{g})$ and the tripeptides $(1 \mu \mathrm{M}-3.3 \mathrm{mM})$ were solved and diluted in the assay buffer. The incubation time was $60 \mathrm{~min}$ at room temperature including 10 measurements with all tripeptide concentrations. 


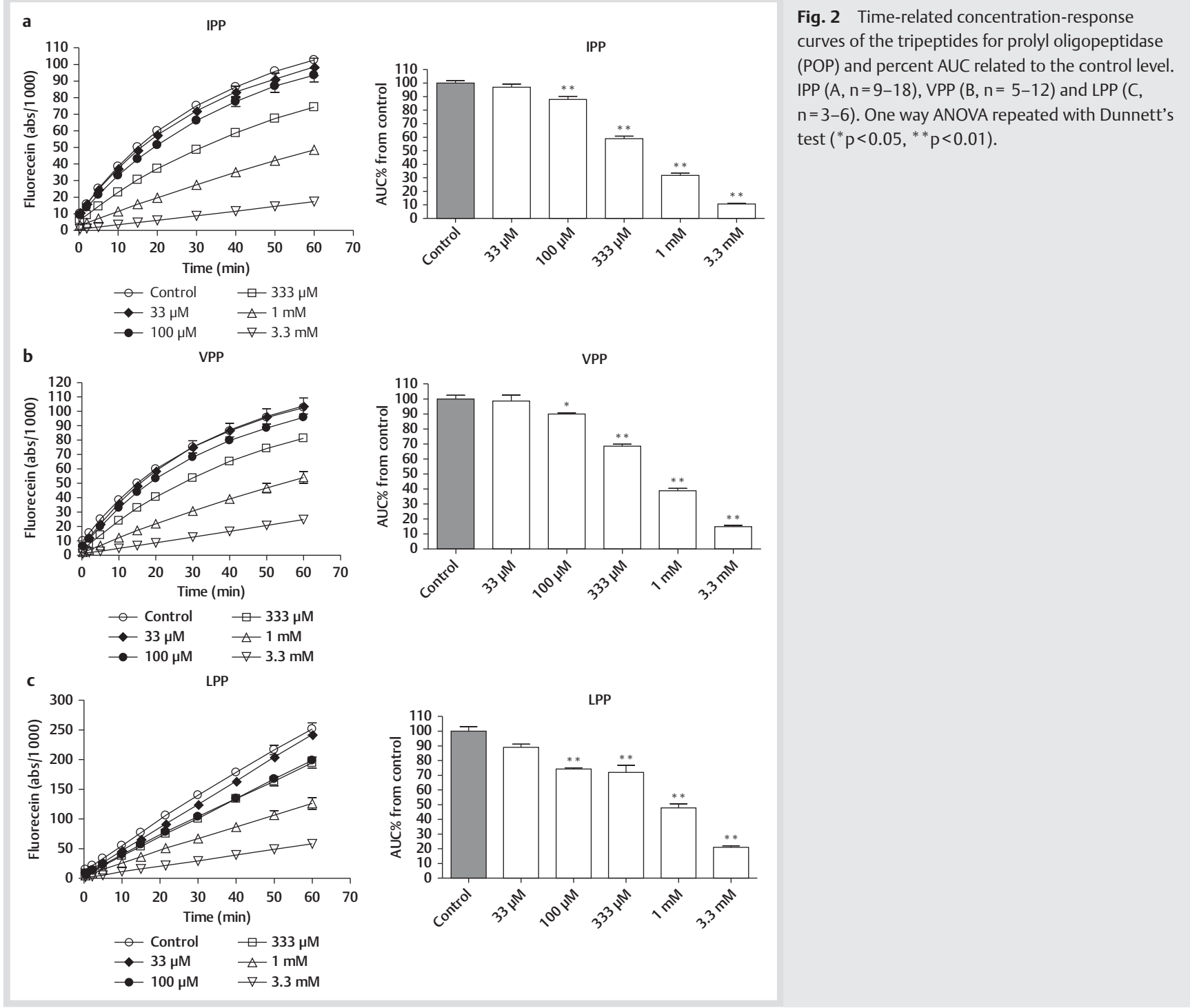

\section{Cyclooxygenase 1 (COX-1) and cyclooxygenase (COX-2) assayes}

The activities of COX- 1 and COX-2 were measured using commercial fluorescence-based assay (COX Fluorescent Inhibitor Screening Assay Kit, Cayman Chemical Company, Ann Arbor, MI, USA). The method includes 2 reactions: COX- 1 and COX-2 form prostaglandin $\mathrm{G}_{2}\left(\mathrm{PGG}_{2}\right)$ from arachidonic acid in the first reaction and in the second reaction $\mathrm{PGG}_{2}$ reacts with 10-acetyl-3.7dihydroxyphenoxazine (ADPH) forming fluorescent product resorufin which can be measured at ex/em $=530 / 590 \mathrm{~nm}$. The tripeptides $(1 \mu \mathrm{M}-3.3 \mathrm{mM})$ were solved and diluted in the assay buffer. The incubation time was $60 \mathrm{~min}$ at room temperature including 10 measurements with all tripeptide concentrations.

\section{Statistical analysis}

The results are presented as mean \pm SEM. Statistical analyses were performed using GraphPad Prism software (version 4.02). One way analysis of variances (ANOVA) followed by Dunnett's multiple comparison tests were used to compare area under a curve (AUC) results. AUC was calculated from the dose-response curves. The activities of the enzymes in different groups were presented as percentage from the control (group without the tripeptide) activity. Difference was considered significant if p-value $<0.05$.

\section{Results \\ $\nabla$}

IPP, VPP and LPP inhibited POP dose-dependently at high micromolar concentrations ( $\bullet$ Fig. 2 ). IC I $_{50}$-values for IPP, VPP and LPP were $486 \pm 95,761 \pm 146$ and $875 \pm 192 \mu \mathrm{M}$ (mean \pm SEM), respectively. The amino acids proline and isoleucine showed no inhibitory effects on POP (data not shown).

Interestingly, all the investigated tripeptides increased the activity of cathepsin $\mathrm{G}(\boldsymbol{\odot} \mathrm{Fig} . \mathbf{3}$ ). Also amino acids proline, isoleucine and arginine elevated cathepsin G activity ( $\bullet$ Fig. 4). Activation was up to $50 \%$ over the control values with all the investigated tripeptides and amino acids. Proline activated cathepsin $\mathrm{G}$ at lower concentrations $(0.1-10 \mu \mathrm{M})$ than IPP, VPP and LPP and amino acids arginine (all $1 \mu \mathrm{M}-3.3 \mathrm{mM})$ and isoleucine $(1-100 \mu \mathrm{M})$.

The tripeptides IPP and VPP showed no inhibition or activation of the other investigated enzymes renin, chymase, NEP, ECE-1, COX-1 and COX-2 $(n=3-11)$ at the concentrations between $1 \mu \mathrm{M}-3.3 \mathrm{mM}$ (Data not shown). 

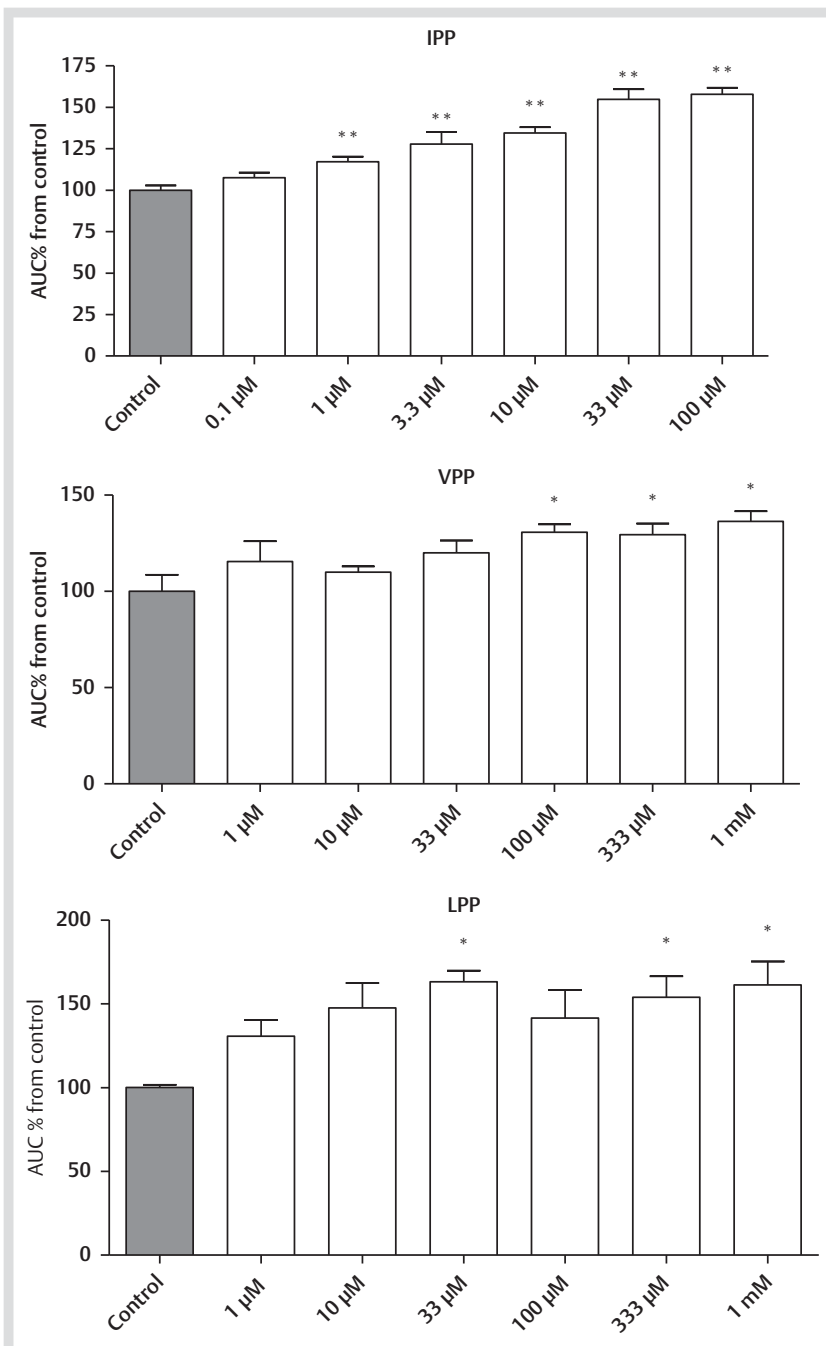

Fig. 3 Percent stimulatory effect of the tripeptides on cathepsin G expressed as the control related AUC (calculated from time-concentration curves) IPP ( $n=3-12), \operatorname{VPP}(n=2-3)$ and LPP $(n=2)$. One way ANOVA repeated with Dunnett's test $\left({ }^{*} p<0.05,{ }^{* *} p<0.01\right)$.

\section{Discussion}

$\nabla$

In the present study, we investigated the effects of milk casein derived tripeptides (IPP, VPP and LPP) on other blood pressure and vascular function regulating enzymes than ACE-1 in vitro using pure commercial enzymes. ACE- 1 was left outside of the analysis because the effect of tripeptides on ACE- 1 has been already tested by us and others. [6,13], Viitala R. et.al unpublished data The tested enzymes were renin, chymase, neutral endopeptidase (NEP), cathepsin $G$ and prolyl oligopeptidase (POP) from RAS and vascular enzymes cyclooxygenase 1 and 2 (COX-1 and COX-2) and endothelin converting enzyme 1 (ECE1). Renin originating from kidney cleaves angiotensinogen to Ang I which is further converted to Ang II by ACE-1. This can also be carried out by chymase and cathepsin G. Cathepsin $G$ is also able to split Ang II directly from angiotensinogen. POP forms Ang-(1-7) from Ang I and Ang II and NEP from Ang I. Also angiotensin converting enzyme 2 (ACE-2) can create Ang-(1-7). ACE1 , NEP and POP participate in the degradation of bradykinin into inactive fragments ( $\odot$ Fig. 1$)[1,2,18,19]$.

In the present study, IPP, VPP and LPP inhibited POP dosedependently. This, however, does not explain their blood pressure lowering effects because POP converts a vasodilatory product Ang(1-7) from Ang I and Ang II. Ang(1-7) is able to stimulate recently describe Mas-receptors ( $\bullet$ Fig. 1 ). Furthermore, relatively high concentrations of the tripeptides were needed for inhibition of POP compared to ACE-1 inhibition $[6,13]$. IC ${ }_{50}$-values were higher than found in plasma after a single dose to the rat [20]. However, IPP seems to accumulate in the tissues and thus in vivo give slight pharmacological effect.

Bradykinin is also a substrate for POP ( $\bullet$ Fig. 1), in addition to Ang I and Ang II at least in in vitro conditions $[19,22,23]$. It may be possible that Ang-(1-7), produced by POP, balances its bradykinin degradation effects in the regulation of blood pressure and vascular function, and therefore POP does not markedly affect blood pressure. However, POP-like activity has been shown to be increased during ACE-1 inhibition in rats [23]. Similarly, also Ang-(1-7) levels are increased during ACE-1 inhibition in rats and in humans [24]. Incubation of mesenteric arteries with IPP improved Ang-(1-7) - and bradykinin-induced mesenteric artery relaxation [5]. This may be due to POP/NEP/ACE-2Ang(1-7)-Mas-reseptor axis and due to ACE-1 inhibition caused by IPP. It is also possible that POP and other Ang-(1-7) forming enzymes, such as NEP and ACE-2, play a physiological role during ACE-1 inhibition or in special pathological situations.

Despite numerous studies $[18,19,21,22,24,25]$, the exact physiological role of POP is unclear. POP cleaves short naturally occurring proline containing peptides on the carboxy side of proline residues. For that reason it may have a role in modulating neuronal peptides and hormones containing proline residues such as substance $P$ and arginine vasopressin. It has been identified as a potential target in cognitive function and in some neurodegenerative disease and POP inhibitors have been utilized for drug development mostly for neurological disorders and as memory enhancers $[24,25]$.

Interestingly, the three tripeptides and all investigated amino acids elevated cathepsin $\mathrm{G}$ activity. The results disagree with a previous study which measured the effects of tripeptides on cathepsin G [13]. In that study, tripeptides had no effect on cathepsin $\mathrm{G}$ but the incubation time was only 10 min while in the present study it was $70 \mathrm{~min}$. It may be possible that the cathepsin $G$ activating effect was not to seen in the short incubation. This is supported by our own in vitro vascular tests [8]. In addition, the effects of amino acids were not tested in our previous study [13]. Concerning in vivo condition amino acids isoleucine and proline stimulated cathepsin G already at such low concentrations which could mean physiological relevance of the findings.

Activation of cathepsin $\mathrm{G}$ cannot explain the decrease of blood pressure and the improvement of vascular function because cathepsin G converts Ang II from angiotensinogen and Ang I ( $\bullet$ Fig. 1). These findings support the assumption that main mechanism for the antihypertensive effect and vascular function improvement by the tripeptides is inhibition of ACE- 1 .

Physiological and pathophysiological compounds can activate cathepsin G. Brecher et al. [26] found that acetaldehyde activated cathepsin $G$ at high millimolar concentrations by ca. $50 \%$ similarly to the peptides and amino acids in the present study. One limitation of the present study is that it was carried out using pure peptides and enzymes in vitro, thus not in physiological conditions. However, these findings show that bioactive, antihypertensive milk-derived peptides are not only ACE-1 inhibitors but have also other effects on the components of RAS. Furthermore, the present study indicates the complexity of the 

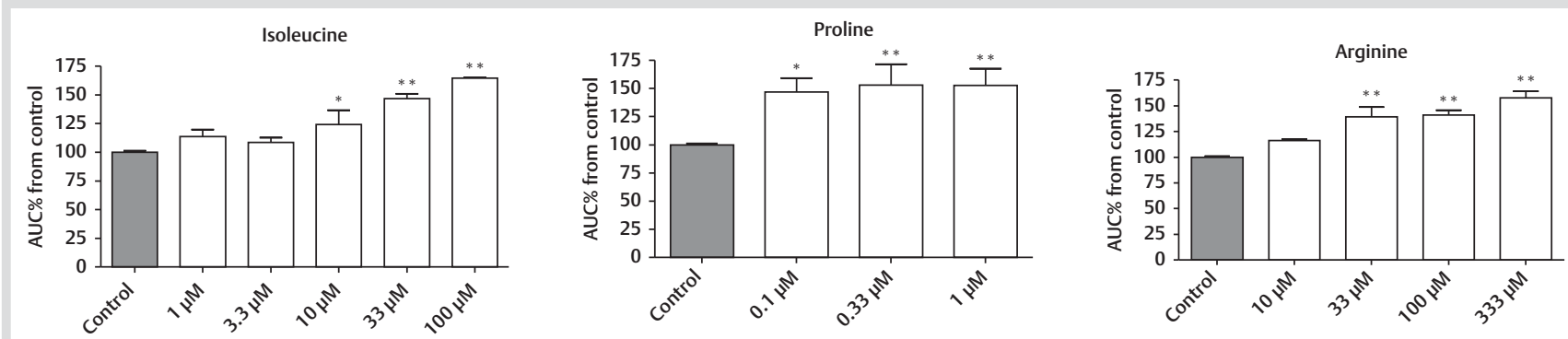

Fig. 4 Percent stimulatory effect of the tripeptides on cathepsin G expressed as the control related AUC (calculated from time-concentration curves) isoleucine $(n=3-8)$, proline $(n=6-14)$ and arginine $(n=2-4)$. One way ANOVA repeated with Dunnett's test $\left({ }^{*} p<0.05,{ }^{* *} p<0.01\right)$.

RAS with its numerous enzymes of which inhibition or activation can change the balance between vasoconstrictive and vasodilating components to unexpected directions. Ex vivo and in vivo experiments are warranted to clarify possible physiological relevance of the findings

\section{Acknowledgements \\ $\nabla$}

This study was supported by the Finnish Funding Agency for Technology and Innovations, TEKES.

\section{Conflict of Interest \\ $\nabla$}

Riitta Korpela was as an employee and Heikki Vapaatalo was consulting for Valio Ltd. at the time when the present study measurements were done.

\section{References}

1 Varagic J, Trask AJ, Jessup JA et al. New angiotensins. J Mol Med 2008; 86: 663-671

2 Fyhrqvist $F$, Saijonmaa 0 . Renin-angiotensin system revisited. J Intern Med 2008; 264: 224-236

3 Katovich MJ, Grobe JL, Raizada MK. Angiotensin-(1-7) as an antihypertensive, antifibrotic target. Curr Hypertens Rep 2008; 10: 227-232

4 Cat AND, Touyz RMA. New look at the renin-angiotensin system- focusing on the vasculature system. Peptides 2011; 32: 2141-2150

5 Ehlers PI, Nurmi L, Turpeinen AM et al. Casein-derived tripeptide Ile-Pro-Pro improves angiotensin-(1-7)- and bradykinin-induced rat mesenteric artery relaxation. Life Sci 2011; 88: 206-211

6 Nakamura Y, Yamamoto N, Sakai $K$ et al. Purification and characterization of angiotensin I-converting enzyme inhibitors from sour milk. J Dairy Sci 1995; 78: 777-783

7 Sipola M, Finckenberg P, Santisteban J et al. Long-term intake of milk peptide attenuates development of hypertension in spontaneously hypertensive rats. J Physiol Pharmacol 2001; 52: 745-754

8 Jäkälä $P$, Jauhiainen $T$, Korpela $R$ et al. Milk protein-derived bioactive tripeptides Ile-Pro-Pro and Val-Pro-Pro protect endothelial function in vitro in hypertensive rats. J Functional Foods 2009; 1: 266-273

9 Jäkälä P, Pere E, Lehtinen $R$ et al. Cardiovascular activity of milk caseinderived tripeptides and plant sterols in spontaneously hypertensive rats. J Physiol Pharmacol 2009; 60: 11-20
10 Pripp AH. Effect of peptides derived from food proteins on blood pressure: a meta-analysis of randomized controlled trials. Food Nutr Res 2008; 32: doi:10.3402/fnr.v52i0.1641

$11 \mathrm{Xu} \mathrm{JY}$, Qin LQ Wang PY et al. Effects of milk tripeptides on blood pressure: a meta-analysis of randomized controlled trials. Nutrition 2008; 24: 933-940

12 Cicero AF, Gerocarni B, Laghi $L$ et al. Blood pressure lowering effects of lactotripeptides assumed as functional food: a meta-analysis of current available clinical trials. J Hum Hypertens 2011; 25: 425-436

13 Lehtinen R, Jauhiainen T, Kankuri $E$ et al. Effects of milk casein-derived tripeptides Ile-Pro-Pro, Val-Pro-Pro, and Leu-Pro-Pro on enzymes processing vasoactive precursors in vitro. Aznelmittelforschung 2010; 60: $182-185$

14 Florentin D, Sassi A, Roques BP. A highly sensitive fluorometric assay for enkephalinase, a neutral metallopeptidase that releases tyrosineglycine-glycine from enkephalins. Anal Biochem 1984; 141: 62-69

15 Atack JR, Suman-Chauhan N, Dawson G et al. In vitro and in vivo inhibition of prolyl endopeptidase. Eur J Pharm 1991; 205: 157-163

16 Stanziola L, Greene LJ, Santos RA. Effect of chronic angiotensin converting enzyme inhibition on angiotensin I and bradykinin metabolism in rats. Am J Hypert 1999; 12: 1021-1029

17 Barrett AJ. Cathepsin G. Meth in Enzym 1981; 80: 561-565

18 Greene LJ, Spadaro AC, Martins A et al. Brain endo-oligopeptidase B: a post-proline cleaving enzyme that inactivates angiotensin I and II. Hypertension 1982; 4: 178-184

19 Ward PE, Bausback HH, Odya CE. Kinin and angiotensin metabolism by purified renal post-proline cleaving enzymes. Biochem Pharmacol 1987: 36: 3187-3193

20 Jauhiainen $T$, Wuolle $K$, Vapaatalo $H$ et al. Oral absoption, tissue distribution and excretion of a radiolabelled analog of a milk-derived antihypertensive peptide, Ile-Pro-Pro, in rats. Int Dairy J 2007; 17 : 1216-1223

21 Koida $M$, Walter $R$. Post-proline cleaving enzyme. Purification of this endopeptidase by affinity chromatography. J Biol Chem 1976; 251: 7593-7599

22 Yoshimoto T, Nishimura T, Takatsuka $N$ et al. Post-proline cleaving enzyme (prolyl endopeptidase) from bovine brain. J Biochem 1983; 94: 1179-1190

23 Stanziola L, Greene LJ, Santos RAS. Effect of chronic angiotensin converting enzyme inhibition on angiotensin I and bradykinin metabolism in rats. Am J Hypertens 1999; 12: 1021-1029

24 Lawandi J, Gerber-Lemaire S, Juillerat-Jeanneret $L$ et al. Inhibitors of prolyl oligopeptidases for the therapy of human disease: Defining diseases and inhibitors. J Med Chem 2010; 53: 3423-3438

25 Carcia-Horsman JA, Männistö PT, Venäläinen JI. On the role of prolyl oligopeptidase on health and disease. Neuropeptides 2007; 41: 1-24

26 Brecher AS, Dubord R. Effect on acetaldehyde upon cathepsin G and chymase. NRAS implications. Dig Dis Sci 2008; 53: 1311-1315 